[0212-7199 (2007) 24: 1; pp 3-11] ANALES DE MEDICINA INTERNA Copyright (C) 2007 ARAN EDICIONES, S.L.

An. Med. INTERNA (Madrid) Vol. 24 , N. ${ }^{\circ} 1$, pp. $3-11,2007$

\title{
Impacto de distintas enfermedades en la calidad de vida relacionada con la salud (CVRS) en una población laboral
}

\author{
M. J. A. LÁINEZ ${ }^{1}$, M. DOMÍNGUEZ ${ }^{2,3}$, J. REJAS ${ }^{4}$, E. ARRIAZA ${ }^{5}$, M. GARCÍA- \\ GARCÍA $^{6}$, G. PALACIOS ${ }^{7}$
}

${ }^{1}$ Servicio de Neurología, Hospital Universitario. Valencia. ${ }^{2}$ Servicio de Neurología. Hospital Puerta de Hierro. Madrid. ${ }^{3}$ Grupo de Neurología del Trabajo. Sociedad Española de Neurología. Madrid. ${ }^{4}$ Departamento de Investigación de Resultados en Salud. Unidad Médica. Pfizer España. Madrid. ${ }^{5}$ Servicio Médico de Empresa Pfizer España. Madrid. ${ }^{6}$ Departamento de Dirección de Proyectos. Biométrica CRO. Barcelona. ${ }^{7}$ Área Neurociencias. Unidad Médica. Pfizer España. Madrid

IMPACT OF SEVERAL ILLNESSES ON HEALTH RELATED QUALITY OF LIFE ON WORKERS

\section{RESUMEN}

Fundamento y objetivo: Realizar un estudio comparativo del grado de impacto de distintas enfermedades en la calidad de vida relacionada con la salud (CVRS) en el medio laboral.

Material y métodos: Un cuestionario incluyendo el cuestionario de CVRS SF-36, fue enviado por correo y cumplimentado por empleados de Pfizer España. Se evaluaron diferencias en el impacto en la CVRS entre sujetos con/sin distintas características, según grado de productividad laboral, y según con/sin antecedentes de distintas enfermedades y notoma/toma actual de tratamiento mediante pruebas estadísticas U MannWhitney y Kruskal-Wallis.

Resultados: En una muestra de 399 sujetos valorables, de 35,8 $\pm 6,5$ años (media \pm desviación típica), un $63,7 \%$ hombres, se observaron peores puntuaciones en el Índice de Salud Física en sujetos con antecentes de alguna enfermedad $(52,6 \pm 5,9$ días versus $55,9 \pm 2,6)$, migraña $(46,2 \pm 5,9$ versus $54,7 \pm 4,1)$, patologías musculoesqueléticas $(49,7 \pm 7,2$ versus $53,9 \pm 5,1)$ y del sistema digestivo $(51,5 \pm 6,6$ versus $54,1 \pm 4,9)$, y toma de algún tratamiento $(50,2 \pm 6,4$ versus $55,0 \pm 4,4) ; \mathrm{p}<0,05$ en todas las comparaciones. En el Índice de Salud Mental se observaron peores puntuaciones en sujetos con antecedentes de alguna enfermedad $(51,4 \pm 7,1$ versus $53,4 \pm 5,9)$, enfermedades del sistema digestivo $(49,9 \pm 8,3$ versus $52,5 \pm 6,3)$ y trastornos mentales $(46,0 \pm 8,8$ versus $52,4 \pm 6,4) ; p<0,05$ en todas las comparaciones.

Conclusiones: El impacto de la enfermedad en la CVRS se asoció al tipo de antecedentes patológicos, siendo la migraña la patología que impacta más negativamente en la Salud Física, y los trastornos mentales en la Salud Mental.

PALABRAS CLAVE: Calidad de vida relacionada con la salud. SF-36. Medicina interna. Estudio transversal. Investigación de resultados en salud.
ABSTRACT

Foundation and objective: To make a comparative study of the degree of impact of different diseases in the health related quality of life (HRQoL) of workers.

Material and methods: A questionnaire including the measure of HRQOL SF-36, was sent by mail and fulfilled by employees of the Pfizer company in Spain. Differences in the impact in HRQoL between subjects with and without several characteristics, with less or more degree of work productivity and with and without antecedents of several diseases and current treatment, were assessed by means of statistical tests of $U$ Mann-Whitney and Kruskal-Wallis.

Results: In a sample of 399 valuable subjects, 35.8 \pm 6.5 years (mean \pm standard deviation), with a $63.7 \%$ of men, worst HRQoL scores in the Physical Summary Component of SF-36 were observed among

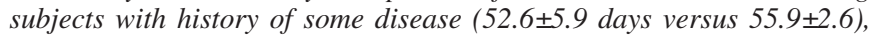
migraine $(46.2 \pm 5.9$ versus $54.7 \pm 4.1)$, musculoskeletal $(49.7 \pm 7.2$ versus $53.9 \pm 5.1)$ and digestive diseases (51.5 \pm 6.6 versus 54.1 \pm 4.9 ), and/or taking some current treatment $(50.2 \pm 6.4$ versus $55.0 \pm 4.4) ; p<0.05$ for all comparisons. In the Physical Summary Component of SF-36 worst HRQoL scores were observed among subjects with history of some disease (51.4 \pm 7.1 versus $53.4 \pm 5.9)$, digestive $(49.9 \pm 8.3$ versus $52.5 \pm 6.3)$ and mental disorders $(46.0 \pm 8.8$ versus $52.4 \pm 6.4) ; p<0.05$ for all comparisons.

Conclusions: The impact of the disease in the HRQoL was associated to the type of pathological antecedents, being migraine that hit more negatively in the Physical Health, and mental disorders in the Mental Health.

KEY WORDS: Health related quality of life. SF-36. Internal medicine. Cross sectional study. Health Outcomes Research.

Láinez MJA, Domínguez M, Rejas J, Arriaza E, García-García M, Palacios G. Impacto de distintas enfermedades en la calidad de vida relacionada con la salud (CVRS) en una población laboral. An Med Interna (Madrid) 2007; 24: 3-11.

\section{INTRODUCCIÓN}

El constante incremento de los costes sociosanitarios en los últimos años es un problema para el cual se están comen- zando a plantear medidas tanto en Estados Unidos (1), como en estados de la Comunidad Europea como el español $(2,3)$. Dada esta creciente restricción de los recursos disponibles, se plantea como una necesidad poder conocer la efectividad y

Trabajo aceptado: 12 de septiembre de 2006

Correspondencia: Javier Rejas Gutiérrez. Departamento de Investigación de Resultados en Salud, Unidad Médica, Pfizer España. Parque Empresarial La Moraleja. Avda. de Europa, 20B. 28108 Alcobendas (Madrid). e-mail: javier.rejas@pfizer.com 
eficiencia de las distintas intervenciones sanitarias tanto desde la perspectiva de la sociedad como de los decisores sanitarios. En este contexto, y de manera creciente, en los estudios que evalúan intervenciones sanitarias se recomienda el uso, como variable de resultado, de la calidad de vida relacionada con la salud (4), habiéndose señalado como un buen predictor del grado de utilización de servicios sanitarios a pesar de que hasta la actualidad todavía la mayoría de sistemas de salud emplean como predictores los diagnósticos registrados por el proveedor (5).

Además de haberse señalado como un útil predictor de utilización de servicios sanitarios, se ha observado que la calidad de vida relacionada con la salud está asociada a otros indicadores de efectividad y eficiencia tales como la productividad laboral relacionada con la salud (6), que resulta ser un indicador de costes indirectos fundamental. El impacto de los problemas de salud sobre la productividad laboral es relevante. Se estima que un $32 \%$ de los sujetos de la población en activo presentan enfermedades crónicas que afectan su trabajo (7). En el contexto de la Medicina Laboral, la estimación de esta afectación que se refleja en forma de pérdidas de productividad laboral relacionadas con la salud, es una información clave a fin de determinar el verdadero impacto de una patología y su tratamiento en una determinada población laboral. Pese a todo ello, existen escasos estudios en que se haya estudiado la relación entre calidad de vida y productividad laboral relacionadas con la salud (6). En los últimos años la Medicina Laboral está intentando adaptar sus objetivos a las cambiantes necesidades del mundo laboral. La continua disminución de enfermedades laborales cuya incidencia está siendo sustituida progresivamente por la creciente presencia de enfermedades crónicas, apunta hacia estrategias de prevención de enfermedades laborales y de protección de la salud de los trabajadores distintas. El seguimiento médico no se debería limitar solamente a valorar la capacitación para realizar el trabajo, sino que debería convertirse en el punto de partida para planificar intervenciones útiles para los trabajadores y la población en general, y el motor de este cambio es la investigación epidemiológica centrada en la mejora de la calidad de la vida.

Por todo ello se planteó la realización del estudio cuyos resultados se presentan en este manuscrito, obtenidos en el contexto de un estudio más amplio de validación de los cuestionarios Migraine Screen Questionnaire (MS-Q) (8) e Índice de Impacto de la enfermedad en la Productividad Laboral (Índice IMPALA) (9) (estudio IMPALA), con el propósito de evaluar el impacto de distintas enfermedades sobre la calidad de vida de una población laboral y su relación con la productividad laboral.

\section{MATERIAL Y MÉTODOS}

\section{DISEÑO Y MUESTRA DEL ESTUDIO}

Se realizó un estudio observacional, transversal, realizado en el ámbito de la Medicina Laboral. Todos los sujetos debían tener 18 o más años de edad, y estar empleados desde hacía un mínimo de 3 meses y a jornada completa en una empresa (Pfizer España). Se excluyeron mujeres embarazadas o de baja maternal y sujetos con imposibilidad de cumplimentar los cuestionarios. Todos los sujetos participantes debían otorgar su consentimiento informado por escrito para participar en el estudio.

\section{CUESTIONARIO DE CALIDAD DE VIDA SF-36}

Se trata de un instrumento de medida de la calidad de vida genérico, que contiene 36 ítems que cubren 8 dimensiones de la salud percibida: función física, rol físico, dolor corporal, salud general, vitalidad, función social, rol emocional y salud mental. Para cada dimensión los ítems se codifican, agregan y transforman en una escala que tiene un recorrido desde 0 (el peor estado de salud) hasta 100 (el mejor estado de salud), como se describe en el manual de puntuación e interpretación del cuestionario (10,11). Adicionalmente, a partir de las puntuaciones de las distintas dimensiones, pueden derivarse las puntuaciones de los Índices de Salud Físico (ISF) y Mental (ISM) de este cuestionario, que también tienen un recorrido de 0 (el peor estado de salud) hasta 100 (el mejor estado de salud).

\section{RECOGIDA DE INFORMACIÓN}

Todos los empleados de la empresa Pfizer España que cumplían criterios de selección fueron contactados por correo para que cumplimentasen un Cuaderno de Recogida de Datos elaborado ad-hoc conjuntamente con el Servicio Médico de Empresa, en el que se recogían los datos de la historia clínica del trabajador y antecedentes de salud de los sujetos participantes, y que incluía el cuestionario de calidad de vida SF-36 descrito en el apartado anterior.

Adicionalmente se evaluó la productividad laboral mediante:

- Índice de IMpacto de la enfermedad en la ProductividAd LAboral (Índice IMPALA). El Índice IMPALA es un cuestionario genérico que consta de 7 ítems que cubren distintos aspectos del impacto laboral de los problemas de salud. Cada ítem debe ser respondido en una escala de respuesta de 1 (impacto laboral durante todo el tiempo o la mayor parte del tiempo de la jornada laboral) hasta 4 (en ningún momento), y existe una quinta opción de respuesta para aquellos casos en que no proceda responder a la pregunta (no aplicable). Puede obtenerse una puntuación total cruda del Índice IMPALA mediante la suma de los puntos obtenidos en los 7 ítems, con un rango de 7 a 28 puntos, que posteriormente se transforma en valores de 0 (peor productividad laboral posible) a 100 (productividad laboral máxima). En el caso de presentar 3 o más ítems "dato perdido" o con respuesta "no aplicable" dicho cuestionario no se consideró valorable. En el caso de presentar "dato perdido" en uno o dos ítems, se imputó el valor medio redondeado del resto de ítems. En el caso de presentar la respuesta "no aplicable" en uno o dos ítems, no se imputó ningún valor en dichos ítems, pero en el momento de transformar la puntuación total cruda a valores entre 0 y 100 se realizó teniendo en cuenta el número de ítems aplicables.

- Número de días equivalentes a días de trabajo perdidos (LWDE)

Se obtiene mediante la siguiente ecuación, $\mathrm{LWDE}_{\mathrm{i}}=\mathrm{W}_{\mathrm{li}}+$ $\mathrm{W}_{2 \mathrm{i}}\left(1-\mathrm{P}_{\mathrm{i}}\right)(12)$, y donde: $\mathrm{W}_{1 \mathrm{i}}$ es el número de días incapaz de trabajar o emprender actividades normales por problemas de salud/crisis de migraña, incluidos dolores de cabeza; $W_{2 i}$ es el número de días en el trabajo con problemas de salud / crisis de migraña; Pi es el porcentaje de efectividad en el trabajo; y $\left(1-\mathrm{P}_{\mathrm{i}}\right)$ es el porcentaje de discapacidad laboral en el trabajo. En el presente estudio el marco temporal de referencia empleado fue el de los 3 últimos meses anteriores al momento de responder los sujetos. 


\section{ANÁLISIS DE LOS DATOS}

En los análisis descriptivos se estimaron la media, la desviación típica y el rango para las variables cuantitativas, y la frecuencia y el porcentaje de sujetos en cada categoría para las variables cualitativas.

Para evaluar las diferencias en calidad de vida entre grupos con distintas características sociodemográficas, distinto grado de productividad laboral, y según presencia/ausencia de antecedentes actuales de patologías, se emplearon las pruebas estadísticas no paramétricas de Mann-Whitney y de KruskalWallis de comparación entre grupos. En segundo lugar, para evaluar la relación entre calidad de vida y productividad laboral se realizaron análisis correlacionales entre las puntuaciones obtenidas con el cuestionario de calidad de vida SF-36 y las obtenidas en el Índice IMPALA y otras medidas de productividad laboral distintas a este Índice incluyendo LWDE, utilizando para ello el coeficiente de correlación de Spearman.

Los análisis realizados cuyos resultados se presentan en el presente trabajo fueron de tipo descriptivo, sin ajustes por posibles factores confusores, a fin de preservar el carácter exploratorio del presente trabajo. Los valores $\mathrm{p}$ referenciados en este manuscrito corresponden a la significación estadística de pruebas con dos colas. Valores inferiores o iguales a 0,05 fueron considerados estadísticamente significativos. Una vez tabulados los datos del estudio y practicado el control de calidad, todos los análisis fueron realizados mediante el paquete estadístico SPSS versión 12.0.

\section{RESULTADOS}

\section{DESCRIPCIÓN DE LA MUESTRA}

La figura 1 describe el flujo de la muestra de 415 sujetos reclutados, de los cuales se obtuvo una muestra total de 399 sujetos valorables que se empleó para todos los análisis, aunque los análisis de las puntuaciones obtenidas con el cuestionario SF-36 y el Índice IMPALA y con el resto de indicadores de productividad laboral, se efectuaron respectivamente con las muestras de sujetos valorables que presentaron el dato en cada uno de estos indicadores.

En la tabla I se describen las principales características sociodemográficas de la muestra total de sujetos del estudio.

La tabla II muestra los resultados del análisis de comparación de las puntuaciones-resumen de los índices de Salud Física y Salud Mental del cuestionario de calidad de vida SF-36 entre grupos con distintas características sociodemográficas, distinto grado de productividad laboral y distintas características según presencia/ausencia de patologías y no toma/toma de tratamiento. Comparando entre estos grupos de sujetos, se pudo observar que se hallaron diferencias estadísticamente significativas en la puntuación obtenida en los Índices de Salud Física y de Salud Mental del Cuestionario SF-36 según sexo, categoría profesional (sólo Salud Mental) y según productividad laboral, observándose menor calidad de vida en sujetos que eran mujeres, con categoría profesional medioalta y con menor productividad laboral (pruebas U de MannWhitney y Kruskal-Wallis: p < 0,05). En la tabla II también se observa peor calidad de vida en términos de Salud Física en sujetos con antecedentes de patologías en general, de migraña, de patologías musculoesqueléticas, del sistema digestivo y de

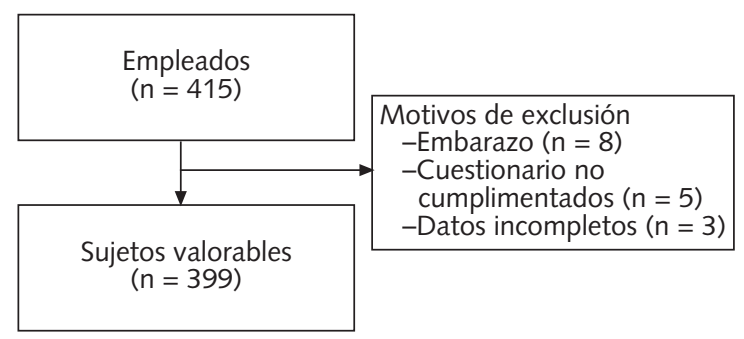

Fig. 1. Esquema de sujetos reclutados, valorables y excluidos.

TABLA I

ADOLESCENTES INGRESADOS EN PEDIATRÍA

\begin{tabular}{lcc}
\hline Características & \multicolumn{2}{c}{ Muestra } \\
& $(\mathrm{n}=399)$ \\
\hline Sexo (n, \%) & 397 & 100,0 \\
Hombre & 253 & 63,7 \\
Mujer & 144 & 36,3 \\
Edad (m, DT) & 35,8 & 6,5 \\
Hombre & 36,8 & 6,4 \\
Mujer & 34,0 & 6,2 \\
Estado civil (n, \%) & 390 & 100,0 \\
Soltero/a & 110 & 28,2 \\
Casado/a & 271 & 69,5 \\
Divorciado/a - separado/a & 9 & 2,3 \\
Índice de Masa Corporal (kg/m2) (m, DT) & 24,7 & 3,5 \\
Hombre & 26,1 & 2,9 \\
Mujer & 22,0 & 2,8 \\
Hábito tabáquico (n, \%) & 371 & 100,0 \\
Fumador & 115 & 31,0 \\
Ex-fumador & 77 & 20,8 \\
No fumador & 179 & 48,2 \\
Categoría profesional (n, \%) & 392 & 100,0 \\
Categoría 1 (sin especialización) & 52 & 13,3 \\
Categoría 2 (especialización baja) & 206 & 52,6 \\
Categoría 3 (especialización medio-alta) & 94 & 24,0 \\
Categoría 4 (especialización alta) & 40 & 10,2 \\
\hline
\end{tabular}

n: número de casos; \%: porcentaje; m: media; DT: Desviación Típica. Categoría 1: ordenanzas, limpiadores, auxiliares, recepcionistas, conductores de vehículos, archivo, grabación de datos, vendedores sin especialización y auxiliares administrativos; categoría 2: vendedores especializados, oficiales administrativos, secretarias y administrativos; categoría 3: supervisores, técnicos y analistas, coordinadores y jefes/médicos de producto; categoría 4: jefes de área/departamento y directores.

toma actual de algún tratamiento (prueba U de Mann-Whitney), versus aquellos sujetos que no presentaban cada una de estas condiciones respectivamente. En términos de Salud Mental solamente se observó peor calidad de vida en sujetos con antecedentes de patologías en general, del sistema digestivo y de trastornos mentales (prueba U de Mann-Whitney), versus aquellos sujetos que no presentaban cada una de estas condiciones respectivamente. 
TABLA II

HALLAZGOS INMUNOHISTOQUÍMICOS

\begin{tabular}{|c|c|c|c|c|c|c|c|c|c|}
\hline & \multicolumn{4}{|c|}{ Índice de salud física } & \multicolumn{4}{|c|}{ Índice de salud mental } \\
\hline & & $n$ & media & $D T$ & $p$ & $n$ & media & $D T$ & $p$ \\
\hline \multirow[t]{2}{*}{ Sexo } & Hombres & 250 & 54,2 & 4,7 & $<0,05^{\mathrm{a}}$ & 250 & 53,4 & 6,0 & $<0,001^{a}$ \\
\hline & Mujeres & 136 & 52,3 & 6,3 & & 136 & 49,3 & 7,5 & \\
\hline \multirow[t]{3}{*}{ Edad } & $\leq 30$ años & 81 & 53,7 & 4,8 & $>0,05^{b}$ & 81 & 50,8 & 7,7 & $>0,05^{b}$ \\
\hline & $>30 y \leq 40$ años & 232 & 53,6 & 5,5 & & 232 & 52,1 & 6,8 & \\
\hline & $>40$ años & 74 & 53,4 & 5,5 & & 74 & 53,1 & 5,4 & \\
\hline \multirow{4}{*}{$\begin{array}{l}\text { Categoría } \\
\text { profesional }\end{array}$} & oría 1 (sin especialización) & 51 & 54,0 & 4,7 & $>0,05^{b}$ & 51 & 53,8 & 4,3 & $<0,05^{b}$ \\
\hline & bría 2 (especialización baja) & 202 & 53,8 & 5,4 & & 202 & 52,2 & 6,7 & \\
\hline & 3 (especialización medio-alta) & 88 & 52,8 & 5,9 & & 88 & 50,0 & 8,4 & \\
\hline & oría 4 (especialización alta) & 40 & 53,1 & 5,0 & & 40 & 52,9 & 4,4 & \\
\hline \multirow[t]{2}{*}{ Índice IMPALA } & ación total > media $(93,2)$ & 232 & 55,1 & 3,7 & $<0,001^{a}$ & 232 & 53,5 & 5,5 & $<0,001^{a}$ \\
\hline & ación total $€$ media $(93,2)$ & 91 & 48,7 & 6,6 & & 91 & 47,9 & 8,1 & \\
\hline \multicolumn{10}{|l|}{$\begin{array}{l}\text { Categorización } \\
\text { de LWDE }\end{array}$} \\
\hline \multirow[t]{3}{*}{ de LWDE } & as en los últimos 3 meses & $\begin{array}{c}226 \\
17\end{array}$ & $\begin{array}{l}55,4 \\
5,5\end{array}$ & $\begin{array}{l}3,5 \\
50\end{array}$ & $<0,001^{b}$ & $\begin{array}{c}226 \\
17\end{array}$ & 53,1 & $\begin{array}{l}5,9 \\
09\end{array}$ & $<0,01^{b}$ \\
\hline & días en los últimos 3 meses & 19 & 51,7 & 5,5 & & $\begin{array}{l}17 \\
19\end{array}$ & $\begin{array}{l}50,5 \\
53,2\end{array}$ & $\begin{array}{l}9,9 \\
6,6\end{array}$ & \\
\hline & ́́as en los últimos 3 meses & 49 & 47,9 & 7,2 & & 49 & 48,7 & 8,4 & \\
\hline \multirow{3}{*}{$\begin{array}{l}\text { Presencia/ausencia de } \\
\text { migraña y/u otras } \\
\text { enfermedades } \\
\text { concomitantes }\end{array}$} & Sí & 274 & 52,6 & 5,9 & & 274 & 51,4 & 7,1 & \\
\hline & No & 114 & 55,9 & 2,6 & $<0,001^{\text {a }}$ & 114 & 53,4 & 5,9 & $<0,01^{\mathrm{a}}$ \\
\hline & No & 114 & 55,9 & 2,6 & & 114 & 53,4 & 5,9 & \\
\hline \multirow[t]{2}{*}{ Migraña } & Sí & 32 & 46,2 & 5,9 & $<0,001^{a}$ & 32 & 50,9 & 6,9 & $>0,05^{\mathrm{a}}$ \\
\hline & No & 237 & 54,7 & 4,1 & & 237 & 52,4 & 6,6 & \\
\hline \multirow[t]{2}{*}{ Lesiones } & Sí & 83 & 54,0 & 5,5 & $>0,05^{a}$ & 83 & 53,5 & 5,3 & $>0,05^{\mathrm{a}}$ \\
\hline & No & 305 & 53,4 & 5,4 & & 305 & 51,6 & 7,1 & \\
\hline \multirow{2}{*}{ Patologías cardíacas } & Sí & 20 & 54,1 & 5,0 & $>0,05^{\mathrm{a}}$ & 20 & 51,2 & 8,6 & $>0,05^{\mathrm{a}}$ \\
\hline & No & 368 & 53,5 & 5,4 & & 368 & 52,1 & 6,7 & \\
\hline \multirow[t]{2}{*}{ Patologías endocrinas } & Sí & 20 & 52,6 & 6,1 & $>0,05^{a}$ & 20 & 51,0 & 8,7 & $>0,05^{a}$ \\
\hline & No & 368 & 53,6 & 5,3 & & 368 & 52,1 & 6,7 & \\
\hline \multirow[t]{2}{*}{ Patologías músculo-esqueléticas } & Sí & 30 & 49,7 & 7,2 & $<0,001^{a}$ & 30 & 50,9 & 8,8 & $>0,05^{a}$ \\
\hline & No & 358 & 53,9 & 5,1 & & 358 & 52,1 & 6,6 & \\
\hline \multirow[t]{2}{*}{ Patologías respiratorias } & Sí & 49 & 52,3 & 6,4 & $>0,05^{a}$ & 49 & 50,4 & 8,3 & $>0,05^{a}$ \\
\hline & No & 339 & 53,7 & 5,2 & & 339 & 52,2 & 6,5 & \\
\hline \multirow[t]{2}{*}{ Sistema digestivo } & Sí & 78 & 51,5 & 6,6 & $<0,01^{\mathrm{a}}$ & 78 & 49,9 & 8,3 & $<0,05^{\mathrm{a}}$ \\
\hline & No & 310 & 54,1 & 4,9 & & 310 & 52,5 & 6,3 & \\
\hline \multirow[t]{2}{*}{ Trastornos mentales } & Sí & 26 & 50,3 & 8,0 & $>0,05^{\mathrm{a}}$ & 26 & 46,0 & 8,8 & $<0,001^{a}$ \\
\hline & No & 362 & 53,8 & 5,1 & & 362 & 52,4 & 6,4 & \\
\hline \multirow[t]{2}{*}{ Toma actual de algún tratamiento } & Sí & 139 & 50,2 & 6,4 & $<0,001^{a}$ & 139 & 51,0 & 7,3 & $>0,05^{\mathrm{a}}$ \\
\hline & No & 108 & 55,0 & 4,4 & & 108 & 51,8 & 7,2 & \\
\hline
\end{tabular}

Mín: mínimo, Máx: máximo, p: nivel de significación.

Categoría 1: ordenanzas, limpiadores, auxiliares, recepcionistas, conductores de vehículos, archivo, grabación de datos, vendedores sin especialización y auxiliares administrativos; categoría 2: vendedores especializados, oficiales administrativos, secretarias y administrativos; categoría 3: supervisores, técnicos y analistas, coordinadores y jefes/médicos de producto; categoría 4: jefes de área/departamento y directores.

a,be encontraron diferencias estadísticamente significativas (en negrita): asegún sexo, Índice IMPALA, presencia/ausencia de enfermedades, y específicamente según presencia/ausencia de migraña, patologías musculoesqueléticas, patologías del sistema digestivo, trastornos mentales, y toma actual de algún tratamiento (prueba U de Mann-Whitney); 'según categoría profesional sólo para el Índice de Salud Mental y según número de días equivalentes a días de trabajo perdidos (LWDE) (prueba de Kruskal-Wallis).

La figura 2 muestra que las puntuaciones obtenidas en los índices de Salud Física y Salud Mental del cuestionario de calidad de vida SF-36 en la muestra del estudio, resultaron superiores a las definidas como valores de referencia en población española para la muestra total y en hombres y mujeres, excepto en el grupo de mujeres en Salud Mental en que las puntuaciones obtenidas en la muestra del estudio no fueron mejores, de forma estadísticamente significativa, que las observadas en población española.

Las tablas III y IV muestran los resultados del análisis de comparación de las distintas dimensiones del cuestionario de calidad de vida SF-36 entre grupos con distintas características. Comparando entre grupos de sujetos con distintas características sociodemográficas y distinto grado de productividad 


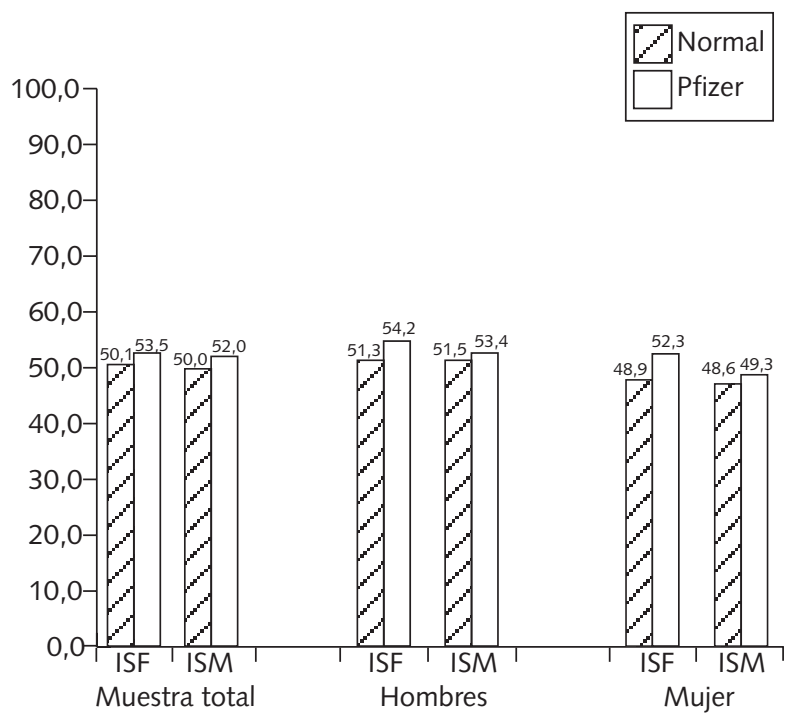

Fig. 2. Puntuaciones en Índices de Salud Física y Mental del cuestionario de calidad de SF-36 con respecto a valores poblacionales de referencia.

laboral, se observó peor calidad de vida en los grupos de sujetos: a) mujeres (para todas las dimensiones); b) jóvenes de 30 o menos años de edad (sólo para dimensión de rol emocional); c) con especialización profesional alta (para dimensiones salud general y vitalidad) y medio-alta (para dimensión rol emocional); d) con peor productividad laboral según puntuación en el Índice IMPALA y según equivalentes a días de trabajo perdidos (LWDE) (para todas las dimensiones) (prueba U de Mann-Whitney para comparación según sexo e Índice IMPALA, y prueba de Kruskal-Wallis para resto de comparaciones). Comparando entre grupos de sujetos según presencia/ausencia de patologías y no toma/toma de tratamiento, se observó peor calidad de vida en los grupos de sujetos con: a) antecedentes de patologías en general (para todas las dimensiones); b) antecedentes de migraña (para todas las dimensiones, excepto en rol emocional); c) sin antecedentes de lesiones (sólo para dimensión de salud mental); d) con antecedentes de patologías musculoesqueléticas (sólo para dimensiones de función social, dolor corporal, y salud general); e) con antecedentes de patologías respiratorias (sólo para dimensiones de rol físico, dolor corporal y rol emocional); f) con antecedentes de patologías del sistema digestivo y de trastornos mentales (para todas las dimensiones); g) toma actual de algún tratamiento (prueba U de Mann-Whitney).

La figura 3 muestra que las puntuaciones obtenidas en las distintas dimensiones del cuestionario de calidad de vida SF36 en la muestra del presente estudio, se mostraron superiores a las observadas como valores de referencia en población española en todas las dimensiones, excepto en función social y dolor en que fueron más similares.

La tabla V muestra los resultados obtenidos en las correlaciones entre el cuestionario de calidad de vida SF-36 y el Índice IMPALA y otras medidas de productividad laboral distintas a este Índice. Se observaron unos coeficientes de correlación de Spearman moderados $(>=0,30 \mathrm{y}<0,70)$ entre el Indice de Salud Mental SF-36 y las medidas de productividad laboral del

TABLA III

PUNTUACIONES EN DIMENSIONES DEL CUESTIONARIO DE CALIDAD DE VIDA SF-36 SEGÚN CARACTERÍSTICAS SOCIODEMOGRÁFICAS, CATEGORÍA PROFESIONAL Y PRODUCTIVIDAD LABORAL

\begin{tabular}{|c|c|c|c|c|c|c|c|c|c|c|c|c|c|c|c|c|c|}
\hline & \multicolumn{3}{|c|}{$P F$} & \multicolumn{2}{|l|}{$R P$} & \multicolumn{2}{|l|}{$B P$} & \multicolumn{2}{|l|}{$\mathrm{GH}$} & \multicolumn{2}{|l|}{ VT } & \multicolumn{2}{|l|}{ SF } & \multirow{2}{*}{$\begin{array}{c}\text { RE } \\
\text { Media }\end{array}$} & \multicolumn{3}{|c|}{$\mathrm{MH}$} \\
\hline & $n$ & Media & DT & Media & DT & Media & DT & Media & DT & Media & DT & Media & DT & & DT & Media & DT \\
\hline \multicolumn{18}{|l|}{$\overline{\operatorname{SexO} 0^{*}}$} \\
\hline Hombres & 253 & 96,0 & 8,8 & 94,0 & 19,3 & 85,1 & 17,9 & 83,9 & 13,2 & 77,7 & 14,5 & 95,5 & 10,3 & 95,3 & 16,4 & 83,9 & 11,8 \\
\hline Mujeres & 144 & 95,5 & 7,2 & 83,2 & 28,9 & 75,4 & 23,6 & 79,8 & 12,7 & 65,5 & 16,3 & 86,4 & 18,4 & 91,1 & 22,1 & 75,1 & 14,8 \\
\hline \multicolumn{18}{|l|}{ Edad** } \\
\hline$\leq 30$ años & 83 & 97,4 & 4,6 & 87,1 & 26,8 & 80,9 & 20,1 & 81,4 & 13,2 & 73,0 & 15,4 & 91,0 & 14,9 & 89,0 & 25,3 & 80,5 & 13,5 \\
\hline$>30 y \leq 40$ años & 239 & 95,7 & 8,6 & 90,2 & 23,8 & 82,2 & 20,7 & 82,6 & 13,1 & 72,7 & 16,8 & 92,1 & 15,1 & 94,5 & 16,9 & 80,8 & 14,1 \\
\hline$>40$ años & 76 & 94,6 & 9,9 & 93,3 & 19,4 & 80,7 & 21,5 & 82,6 & 13,3 & 75,6 & 15,3 & 94,1 & 11,5 & 96,9 & 14,7 & 80,9 & 12,2 \\
\hline \multicolumn{18}{|l|}{ Categoría profesional** } \\
\hline Categoría 1 (sin especialización) & 52 & 97,8 & 5,2 & 94,2 & 16,9 & 84,4 & 19,5 & 83,1 & 11,7 & 77,7 & 12,5 & 94,2 & 11,5 & 98,7 & 6,5 & 83,5 & 11,2 \\
\hline Categoría 2 (especialización baja) & 206 & 95,5 & 9,0 & 90,2 & 24,0 & 81,3 & 21,3 & 84,5 & 12,1 & 75,0 & 16,1 & 92,9 & 13,7 & 93,3 & 20,2 & 80,8 & 14,2 \\
\hline Categoría 3 (especialización medio-alta) & 94 & 94,9 & 9,0 & 87,5 & 27,1 & 79,2 & 21,5 & 79,3 & 14,2 & 67,2 & 17,4 & 88,6 & 18,5 & 90,5 & 21,9 & 78,7 & 14,1 \\
\hline $\begin{array}{l}\text { Categoría } 4 \text { (especialización alta) } \\
\text { indice IMPALA* }\end{array}$ & 40 & 96,6 & 5,2 & 90,6 & 22,4 & 84,4 & 17,4 & 78,2 & 15,3 & 72,4 & 14,9 & 94,4 & 9,4 & 98,3 & 7,4 & 81,4 & 11,1 \\
\hline Puntuación total $\leq$ media $(93,2)$ & 92 & 92,0 & 10,9 & 68,0 & 34,2 & 61,1 & 19,9 & 76,0 & 14,0 & 61,6 & 16,7 & 79,5 & 19,9 & 85,1 & 27,7 & 72,0 & 14,5 \\
\hline Puntuación total > media $(93,2)$ & 239 & 97,2 & 6,2 & 96,6 & 14,5 & 87,8 & 15,5 & 84,5 & 11,8 & 77,4 & 13,5 & 96,8 & 8,3 & 96,4 & 14,6 & 84,0 & 11,6 \\
\hline \multicolumn{18}{|l|}{ Categorización de LWDE** } \\
\hline 0 días en los últimos 3 meses & 233 & 97,3 & 6,5 & 97,7 & 10,8 & 87,9 & 16,0 & 85,2 & 11,4 & 77,4 & 13,9 & 96,0 & 9,7 & 95,5 & 17,3 & 83,3 & 12,4 \\
\hline$>0-<1$ días en los últimos 3 meses & 19 & 93,9 & 9,3 & 91,7 & 21,0 & 73,9 & 24,1 & 79,8 & 16,2 & 72,9 & 19,2 & 86,2 & 23,2 & 90,7 & 22,3 & 78,8 & 16,3 \\
\hline$\geq 1-<2$ días en los últimos 3 meses & 20 & 97,3 & 3,8 & 86,3 & 27,5 & 70,6 & 18,8 & 82,3 & 12,0 & 69,4 & 15,2 & 94,4 & 8,6 & 95,0 & 16,3 & 83,8 & 11,7 \\
\hline$\geq 2$ días en los últimos 3 meses & 49 & 91,1 & 11,6 & 60,7 & 38,5 & 65,2 & 23,4 & 75,3 & 14,2 & 60,6 & 18,0 & 78,8 & 21,4 & 87,8 & 24,2 & 73,2 & 13,9 \\
\hline
\end{tabular}

Función Física (PF) (0-100); Rol Físico (RP) (0-100); Dolor Corporal (BP) (0-100); Salud General (GH) (0-100); Vitalidad (VT) (0-100); Función Social (SF) (0-100); Rol Emocional (RE) (0-100); Salud Mental (MH) (0-100) LWDE: número de días equivalentes a días de trabajo perdidos, n: número de casos, DT: Desviación Típica, Mín: mínimo, Máx: máximo, \%: porcentaje. ${ }^{*}{ }^{*}$ * Se encontraron diferencias estadísticamente significativas (en negrita): asegún sexo e Índice IMPALA, para todas las dimensiones (prueba U de Mann-Whitney); bsegún edad para la dimensión de rol emocional, según categoría profesional para las dimensiones de salud general, vitalidad y rol emocional y según número de días equivalentes a días de trabajo perdidos (LWDE) para todas las dimensiones (prueba de Kruskal-Wallis). 
TABLA IV

PUNTUACIONES EN DIMENSIONES DEL CUESTIONARIO DE CALIDAD DE VIDA SF-36 SEGÚN ANTECEDENTES PATOLÓGICOS Y TOMA DE ALGÚN TRATAMIENTO

\begin{tabular}{|c|c|c|c|c|c|c|c|c|c|c|c|c|c|c|c|c|c|}
\hline & $n$ & $\begin{array}{c}\text { PF } \\
\text { Media }\end{array}$ & DT & $\begin{array}{c}R P \\
\text { Media } \\
\end{array}$ & DT & $\begin{array}{c}\text { BP } \\
\text { Media }\end{array}$ & DT & $\begin{array}{c}\mathrm{CH} \\
\text { Media }\end{array}$ & DT & $\begin{array}{c}\text { VT } \\
\text { Media } \\
\end{array}$ & DT & $\begin{array}{c}\text { SF } \\
\text { Media }\end{array}$ & DT & $\begin{array}{c}R E \\
\text { Media } \\
\end{array}$ & DT & $\begin{array}{c}\text { MH } \\
\text { Media } \\
\end{array}$ & DT \\
\hline \multicolumn{18}{|c|}{$\begin{array}{l}\text { Presencia/ausencia de migraña } \\
\text { y/u otras enfermedades } \\
\text { concomitantes }^{\mathrm{a}}\end{array}$} \\
\hline Sí & 284 & 95,0 & 9,3 & 86,5 & 27,1 & 77,8 & 21,7 & 80,7 & 13,6 & 71,2 & 16,8 & 90,3 & 15,7 & 92,5 & 20,7 & 79,3 & 13,8 \\
\hline No & 115 & 98,0 & 3,7 & 99,1 & 5,7 & 90,8 & 14,2 & 86,3 & 11,2 & 78,9 & 13,5 & 96,8 & 9,2 & 97,1 & 12,1 & 84,5 & 12,4 \\
\hline \multicolumn{18}{|l|}{ Migrañ̃a ${ }^{a}$} \\
\hline Migraña & 35 & 92,1 & 10,3 & 64,5 & 32,6 & 57,2 & 21,9 & 75,7 & 16,6 & 62,1 & 17,3 & 80,4 & 20,2 & 94,1 & 17,4 & 76,6 & 13,8 \\
\hline No migraña & 240 & 96,9 & 6,7 & 93,9 & 19,3 & 86,4 & 18,2 & 84,0 & 12,1 & 74,8 & 15,9 & 94,5 & 12,9 & 94,5 & 18,0 & 82,4 & 12,1 \\
\hline \multicolumn{18}{|l|}{ Lesiones $^{\mathrm{a}}$} \\
\hline Presencia & 87 & 95,8 & 10,0 & 93,1 & 18,2 & 84,1 & 18,6 & 84,0 & 13,4 & 76,1 & 13,8 & 95,4 & 9,4 & 95,8 & 15,9 & 84,0 & 10,4 \\
\hline Ausencia & 312 & 95,8 & 7,7 & 89,3 & 25,0 & 80,9 & 21,2 & 81,9 & 13,1 & 72,7 & 16,8 & 91,3 & 15,5 & 93,3 & 19,4 & 79,9 & 14,2 \\
\hline \multicolumn{18}{|l|}{ Patologías cardiacas } \\
\hline Presencia & 22 & 94,3 & 9,4 & 91,7 & 19,9 & 82,0 & 18,4 & 81,6 & 14,2 & 68,0 & 18,6 & 88,6 & 21,4 & 90,5 & 26,1 & 76,9 & 19,4 \\
\hline Ausencia & 377 & 95,9 & 8,2 & 90,1 & 23,9 & 81,6 & 20,8 & 82,4 & 13,1 & 73,7 & 16,1 & 92,4 & 13,9 & 94,0 & 18,2 & 81,0 & 13,2 \\
\hline \multicolumn{18}{|l|}{ Patologías endocrinas } \\
\hline Presencia & 20 & 91,0 & 13,9 & 93,8 & 22,8 & 77,6 & 19,7 & 77,6 & 15,3 & 70,7 & 16,2 & 92,5 & 13,1 & 90,0 & 21,9 & 78,4 & 14,1 \\
\hline Ausencia & 379 & 96,1 & 7,8 & 90,0 & 23,8 & 81,8 & 20,7 & 82,6 & 13,0 & 73,6 & 16,2 & 92,2 & 14,5 & 94,0 & 18,5 & 80,9 & 13,6 \\
\hline \multicolumn{18}{|c|}{ Patologías musculoesqueléticas } \\
\hline Presencia & 30 & 86,7 & 15,0 & 86,7 & 26,0 & 68,2 & 21,3 & 73,8 & 13,4 & 68,4 & 18,7 & 88,3 & 17,3 & 88,3 & 28,8 & 77,2 & 14,3 \\
\hline Ausencia & 369 & 96,6 & 6,9 & 90,5 & 23,5 & 82,7 & 20,3 & 83,0 & 12,9 & 73,8 & 16,0 & 92,5 & 14,2 & 94,3 & 17,6 & 81,1 & 13,5 \\
\hline \multicolumn{18}{|l|}{ Patologías respiratorias ${ }^{\mathrm{a}}$} \\
\hline Presencia & 51 & 95,3 & 7,5 & 83,8 & 27,8 & 75,9 & 20,5 & 78,9 & 14,5 & 69,7 & 16,5 & 89,7 & 15,4 & 89,5 & 23,6 & 78,3 & 14,6 \\
\hline Ausenci & 348 & 95,9 & 8,3 & 91,1 & 23,0 & 82,4 & 20,6 & 82,8 & 12,9 & 73,9 & 16,1 & 92,6 & 14,3 & 94,5 & 17,8 & 81,2 & 13,4 \\
\hline \multicolumn{18}{|l|}{ Sistema digestivoa } \\
\hline Presencia & 82 & 93,9 & 9,4 & 81,4 & 30,3 & 72,2 & 22,5 & 78,0 & 13,7 & 68,0 & 18,4 & 87,7 & 18,5 & 88,5 & 24,8 & 75,2 & 16,2 \\
\hline Ausencia & 317 & 96,3 & 7,8 & 92,4 & 21,2 & 84,0 & 19,5 & 83,5 & 12,8 & 74,8 & 15,4 & 93,4 & 13,0 & 95,2 & 16,6 & 82,2 & 12,5 \\
\hline \multicolumn{18}{|l|}{ Trastornos mentaless } \\
\hline Presencia & 27 & 91,3 & 12,4 & 67,9 & 32,0 & 67,8 & 21,1 & 74,9 & 13,1 & 59,6 & 16,4 & 82,9 & 19,0 & 80,8 & 31,5 & 67,1 & 11,5 \\
\hline Ausencia & 372 & 96,2 & 7,8 & 91,7 & 22,3 & 82,6 & 20,3 & 82,8 & 13,0 & 74,4 & 15,8 & 92,9 & 13,8 & 94,7 & 17,2 & 81,8 & 13,2 \\
\hline \multicolumn{18}{|c|}{ Toma actual de algún tratamiento ${ }^{a}$} \\
\hline Sin tratamic & 111 & 96,8 & 5,8 & 92,3 & 22,1 & 86, & 17,5 & 85,1 & 11,5 & 75,7 & 16,3 & 93,2 & 14,4 & 92,5 & 20,9 & 81,5 & 14,4 \\
\hline Con tratamiento actual & 144 & 92,8 & 11,5 & 79,3 & 31,0 & 69,6 & 22,3 & 77,1 & 14,5 & 67,2 & 17,3 & 87,2 & 17,1 & 91,9 & 21,2 & 76,7 & 13,8 \\
\hline
\end{tabular}

Función Física (PF) (0-100); Rol Físico (RP) (0-100); Dolor Corporal (BP) (0-100); Salud General (GH) (0-100); Vitalidad (VT) (0-100); Función Social (SF) (0-100); Rol Emocional (RE) (0-100); Salud Mental (MH) (0-100). a'Se encontraron diferencias estadísticamente significativas (en negrita) según presencia/ausencia de enfermedades, y específicamente según presencia/ausencia de migraña, de lesiones, de patologías musculoesqueléticas, de patologías respiratorias, de patologías del sistema digestivo y de trastornos mentales, y según toma actual de algún tratamiento (prueba $U$ de MannWhitney).

Índice IMPALA $(\mathrm{r}=0,57)$, días de trabajo con síntomas $(\mathrm{r}=-$ $0,44)$, porcentaje del rendimiento $(\mathrm{r}=0,41)$ y número de días equivalentes a días de trabajo perdidos LWDE ( $r=-0,48)$. También se observaron coeficientes de correlación de Spearman moderados entre el Indice de Salud Física SF-36 y las medidas de productividad laboral del Índice IMPALA $(r=0,45)$, porcentaje del rendimiento $(\mathrm{r}=0,35)$ y número de días equivalentes a días de trabajo perdidos LWDE $(r=-0,32)$.

\section{DISCUSIÓN}

\section{CALIDAD DE VIDA EN POBLACIÓN LABORAL SEGÚN CARACTERÍSTICAS SOCIODEMOGRÁFICAS Y PRODUCTIVIDAD LABORAL}

En el presente estudio, se observó peor calidad de vida relacionada con la salud en sujetos mujeres, con categoría profesional medio-alta y que presentaban menor productividad laboral según puntuación del Índice IMPALA y según número de días equivalentes a días de trabajo perdidos (LWDE), lo cual resulta coherente con lo observado en otros trabajos con respecto a las diferencias por sexo (13) y según productividad laboral (6).

\section{CALIDAD DE VIDA EN POBLACIÓN LABORAL SEGÚN PATOLOGÍAS Y TOMA DE ALGÚN TRATAMIENTO}

Por patologías, los sujetos que presentaron peor calidad de vida en términos de Salud Física fueron los sujetos con antecedentes de migraña, y en términos de Salud Mental, los sujetos con antecedentes de trastornos mentales. En particular, se observó un mayor impacto en la calidad de vida en los sujetos con antecedentes de alguna enfermedad en general, alguna enfermedad musculoesquelética (sólo en términos de 

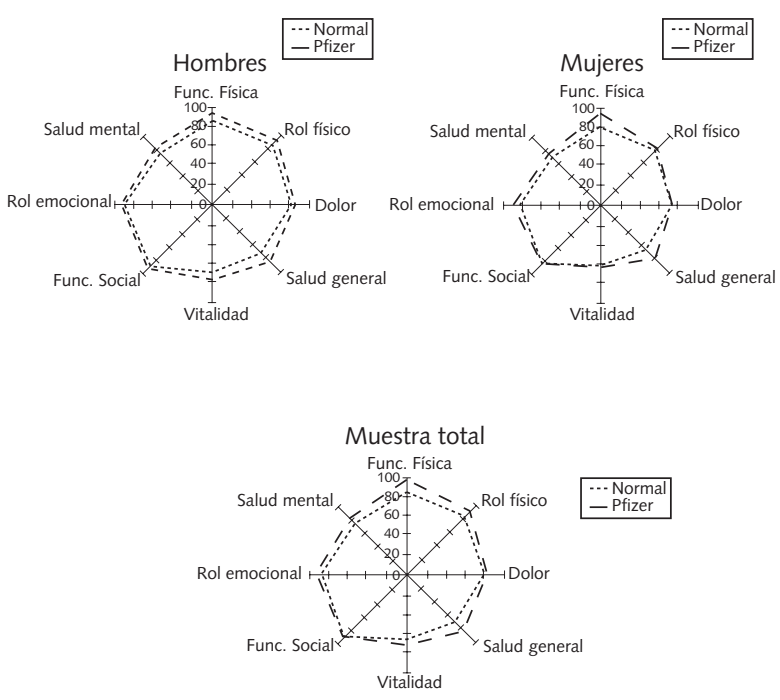

Fig. 3. Puntuaciones en dimensiones del cuestionario de calidad de vida SF-36 con respecto a valores poblacionales de referencia.

Salud Física), del sistema digestivo, trastorno mental (sólo en términos de Salud Mental), o migraña (sólo en términos de Salud Física), o que estaban recibiendo algún tratamiento (sólo en términos de Salud Física), versus a sujetos que no presentaban cada uno de estos antecedentes ni tampoco esta- ban recibiendo ningún tratamiento. Estos resultados son congruentes con los obtenidos en estudios previos, en que se ha descrito el impacto en la calidad de vida de patologías en general (14), musculoesqueléticas (15), digestivas (16), trastornos mentales (17), y migraña (18).

Sin embargo, los resultados obtenidos presentan una aparente contradicción con los mostrados en otros estudios previos, al no haberse observado una peor calidad de vida en sujetos con antecedentes de patologías cardíacas y endocrinas, para ninguna dimensión, y tampoco en sujetos con antecedentes de enfermedades respiratorias para ninguna dimensión, excepto rol físico, dolor corporal y rol emocional. Este resultado es aparentemente contradictorio frente a otros estudios en que sí se ha observado el impacto de la enfermedad en la calidad de vida de los pacientes con patologías cardíacas $(14)$, endocrinas $(14,19)$ y respiratorias (20). Esta discrepancia debe ser atribuida parcialmente al reducido tamaño de los grupos de sujetos con prevalencia de antecedentes de patologías cardíacas y endocrinas en el presente estudio ( $n=20$ para ambos tipos de patología), y en el caso de las patologías cardíacas y respiratorias, al hecho de que la mayoría del total de pacientes con patologías cardíacas presentaban hipertensión $(n=15 / 22,68,2 \%)$, y la mayoría del total de pacientes con antecedentes de patologías respiratorias presentaban asma ( $\mathrm{n}=28 / 51,54,9 \%)$, pneumonía o gripe $(\mathrm{n}=16 / 51,31,4 \%)$, y/o manifestaciones alérgicas $(\mathrm{n}=12 / 51,23,5 \%)$. Pese a esta aparente limitación a considerar en la interpretación de los resultados obtenidos, que puede estar sesgándolos parcialmente, infravalorando el impacto en calidad de vida de patologías agudas

\section{TABLA V}

RELACIÓN ENTRE CALIDAD DE VIDA Y PRODUCTIVIDAD LABORAL: CORRELACIONES ENTRE PUNTUACIONES EN LOS CUESTIONARIOS DE CALIDAD DE VIDA SF-36, E ÍNDICE IMPALA Y OTRAS MEDIDAS DE PRODUCTIVIDAD LABORAL

\begin{tabular}{|c|c|c|c|c|c|c|}
\hline & & $\begin{array}{l}\text { Índice } \\
\text { IMPALA }\end{array}$ & $\begin{array}{c}\text { Días en que } \\
\text { se falta }\end{array}$ & $\begin{array}{l}\text { Días con } \\
\text { síntomas }\end{array}$ & $\begin{array}{c}\% \\
\text { rendimiento }\end{array}$ & $\angle W D E$ \\
\hline$S F-36 P F$ & $\begin{array}{l}\text { Coef. Corr. } \\
\text { Sig. }\end{array}$ & $\begin{array}{c}0,32 \\
<0,001\end{array}$ & $\begin{array}{l}-0,15 \\
0,044\end{array}$ & $\begin{array}{c}-0,28 \\
<0,001\end{array}$ & $\begin{array}{c}0,21 \\
0,004\end{array}$ & $\begin{array}{c}-0,28 \\
<0,001\end{array}$ \\
\hline$S F-36 R P$ & $\begin{array}{l}\text { Coef. Corr. } \\
\text { Sig. }\end{array}$ & $\begin{array}{c}0,63 \\
<0,001\end{array}$ & $\begin{array}{c}-0,28 \\
<0,001\end{array}$ & $\begin{array}{c}-0,53 \\
<0,001\end{array}$ & $\begin{array}{c}0,50 \\
<0,001\end{array}$ & $\begin{array}{c}-0,57 \\
<0,001\end{array}$ \\
\hline$S F-36 B P$ & $\begin{array}{l}\text { Coef. Corr. } \\
\text { Sig. }\end{array}$ & $\begin{array}{c}0,58 \\
<0,001\end{array}$ & $\begin{array}{l}-0,13 \\
0,085\end{array}$ & $\begin{array}{c}-0,45 \\
<0,001\end{array}$ & $\begin{array}{c}0,40 \\
<0,001\end{array}$ & $\begin{array}{c}-0,43 \\
<0,001\end{array}$ \\
\hline$S F-36 G H$ & $\begin{array}{l}\text { Coef. Corr. } \\
\text { Sig. }\end{array}$ & $\begin{aligned} & 0,37 \\
< & 0,001\end{aligned}$ & $\begin{array}{l}-0,13 \\
0,077\end{array}$ & $\begin{array}{c}-0,31 \\
<0,001\end{array}$ & $\begin{aligned} & 0,27 \\
< & 0,001\end{aligned}$ & $\begin{array}{c}-0,29 \\
<0,001\end{array}$ \\
\hline$S F-36 V T$ & $\begin{array}{l}\text { Coef. Corr. } \\
\text { Sig. }\end{array}$ & $\begin{aligned} & 0,55 \\
< & 0,001\end{aligned}$ & $\begin{array}{l}-0,15 \\
0,048\end{array}$ & $\begin{array}{c}-0,40 \\
<0,001\end{array}$ & $\begin{array}{c}0,41 \\
<0,001\end{array}$ & $\begin{array}{c}-0,44 \\
<0,001\end{array}$ \\
\hline$S F-36 S F$ & $\begin{array}{l}\text { Coef. Corr. } \\
\text { Sig. }\end{array}$ & $\begin{array}{c}0,62 \\
<0,001\end{array}$ & $\begin{aligned} & -0,26 \\
< & 0,001\end{aligned}$ & $\begin{array}{c}-0,43 \\
<0,001\end{array}$ & $\begin{array}{c}0,43 \\
<0,001\end{array}$ & $\begin{array}{c}-0,49 \\
<0,001\end{array}$ \\
\hline$S F-36 R E$ & $\begin{array}{l}\text { Coef. Corr. } \\
\text { Sig. }\end{array}$ & $\begin{array}{c}0,29 \\
<0,001\end{array}$ & $\begin{array}{c}0,07 \\
0,330\end{array}$ & $\begin{array}{c}-0,26 \\
<0,001\end{array}$ & $\begin{array}{c}0,20 \\
0,007\end{array}$ & $\begin{array}{l}-0,21 \\
0,005\end{array}$ \\
\hline$S F-36 \mathrm{MH}$ & $\begin{array}{l}\text { Coef. Corr. } \\
\text { Sig. }\end{array}$ & $\begin{array}{c}0,49 \\
<0,001\end{array}$ & $\begin{array}{l}-0,04 \\
0,626\end{array}$ & $\begin{array}{c}-0,34 \\
<0,001\end{array}$ & $\begin{array}{c}0,40 \\
<0,001\end{array}$ & $\begin{array}{c}-0,37 \\
<0,001\end{array}$ \\
\hline SF-36 ISF & $\begin{array}{l}\text { Coef. Corr. } \\
\text { Sig. }\end{array}$ & $\begin{aligned} & 0,57 \\
< & 0,001\end{aligned}$ & $\begin{aligned} & -0,27 \\
< & 0,001\end{aligned}$ & $\begin{aligned} & -0,44 \\
< & 0,001\end{aligned}$ & $\begin{array}{c}0,41 \\
<0,001\end{array}$ & $\begin{aligned} & -0,48 \\
< & 0,001\end{aligned}$ \\
\hline SF-36ISM & $\begin{array}{l}\text { Coef. Corr. } \\
\text { Sig. }\end{array}$ & $\begin{array}{c}0,45 \\
<0,001\end{array}$ & $\begin{array}{l}-0,04 \\
0,623\end{array}$ & $\begin{array}{c}-0,29 \\
<0,001\end{array}$ & $\begin{array}{c}0,35 \\
<0,001\end{array}$ & $\begin{array}{c}-0,32 \\
<0,001\end{array}$ \\
\hline
\end{tabular}

LWDE (número de días equivalentes a días de trabajo perdidos); PF (Función Física), RP (Rol Físico), BP (Dolor Corporal), GH (Salud General), VT (Vitalidad), SF (Función Social), RE (Rol Emocional), MH (Salud Mental), ISF (Índice de Salud Física), ISM (Índice de Salud Mental); correlaciones calculadas mediante rho de Spearman, tomando la misma muestra para todos los pares $(n=184) ; r$ : coeficiente de correlación; p: significación bilateral; para los valores de significación $p=0,0000$, se procedió a substituir por $p<0,001$. 
sin episodios en los 3 últimos meses, el impacto asociado a las distintas patologías estudiadas resultó comparable al observado en los otros escasos estudios existentes. Finalmente, también debe destacarse como otra aparente contradicción la mejor puntuación obtenida en la dimensión de salud mental en los pacientes con antecedentes de lesiones, resultado que sin embargo debe atribuirse a la relación esperable entre ejercicio físico y mayor presencia de lesiones y mayor salud mental.

\section{CALIDAD DE VIDA EN LA POBLACIÓN LABORAL EN ESTUDIO Y EN LA POBLACIÓN GENERAL ESPAÑOLA}

La calidad de vida observada en la muestra del estudio, resultó en general superior a la observada en población española para la muestra total y en hombres y mujeres, tanto para las puntuaciones-resumen de los Índices de Salud Física y Mental del cuestionario de calidad de vida SF-36 como para las puntuaciones de todas las dimensiones, resultado que debe su explicación a las distintas condiciones laborales y sociales de la muestra del estudio y la población española en general. Adicionalmente, debe destacarse que esta mejor calidad de vida de la muestra estudiada presenta una excepción en el grupo de mujeres para el Indice de Salud Mental, en que las puntuaciones obtenidas en la muestra del estudio fueron similares a las observadas en población española. Este resultado puede atribuirse al hecho de que en la muestra del estudio todas las mujeres por definición se encontraban en activo, mientras que en población española una proporción relevante de mujeres no trabaja.

\section{CALIDAD DE VIDA Y PRODUCTIVIDAD LABORAL RELACIONADAS CON LA SALUD: INSTRUMENTOS DE MEDIDA Y POSIBLES LIMITACIONES}

Uno de los problemas de la evaluación de resultados sanitarios ("outcomes") tales como la calidad de vida y la productividad laboral, es la elección de medidas adecuadamente factibles y válidas para su medida. En el presente estudio para la evaluación de la calidad de vida se empleó el Cuestionario de Calidad de Vida SF-36, instrumento genérico que ha demostrado adecuadas propiedades psicométricas (13), y para el cual se dispone de valores poblacionales de referencia que permiten la comparación de puntuaciones entre distintas poblaciones (13). Para la cuantificación de las pérdidas de productividad laboral entendidas como costes directos existen diversas medidas, de entre las cuales actualmente la más habitualmente aceptada y empleada es la de los días equivalentes a días de trabajo perdidos (LWDE). Por otra parte, existen diversos instrumentos, tan- to genéricos como específicos, para la medida de las pérdidas de productividad laboral entendidas como costes indirectos, tal y como señalan dos recientes revisiones al respecto $(21,22)$. En este sentido debe destacarse también que las medidas de productividad laboral empleadas en este estudio, Índice IMPALA (9) y LWDE, son medidas factibles, fiables y válidas. La elección de instrumentos de medida adecuados apoya la validez de los resultados obtenidos en el presente estudio.

\section{CALIDAD DE VIDA Y PRODUCTIVIDAD LABORAL RELACIONADAS CON LA SALUD: OTROS FACTORES}

En este estudio se observan asociaciones entre el impacto en la calidad de vida y en la productividad laboral de distintas enfermedades, observándose mayor relación del grado de productividad laboral con la calidad de vida en términos de Salud Física. Sin embargo, estas asociaciones deben interpretarse considerando la limitación que supone haber sido obtenidas en un estudio de diseño transversal, de tal modo que el sentido de las posibles relaciones de causalidad en ningún caso puede determinarse. Más allá de esta limitación, y de la complejidad intrínseca de la relación entre calidad de vida y productividad (y actividad) laboral relacionadas con la salud, debe considerarse la complejidad de las relaciones de la calidad de vida con otros posibles factores que, al no haber sido controlados en el presente estudio, suponen una limitación a considerar en la interpretación de los resultados obtenidos. Entre estos posibles factores estarían incluídos la adherencia al tratamiento $(23,24)$, estrategias de apoyo al paciente como psicoeducación (25), presencia de dolor (26), estilos de vida (27), estilo de afrontamiento de problemas y disponibilidad de recursos psicosociales (28), y características asociadas a la propia actividad laboral, de los cuales se han señalado sus efectos sobre la calidad de vida relacionada con la salud de los sujetos, tanto positivos $(29,30)$ como negativos (31).

\section{FUTURAS INVESTIGACIONES}

Para futuros trabajos queda pendiente completar la evaluación de la calidad de vida y de su relación con la productividad laboral con la réplica del presente trabajo en otras poblaciones distintas a la estudiada. Asimismo, queda pendiente la realización de futuras investigaciones con diseños prospectivos más complejos que permitan establecer en el entorno laboral, el sentido de las relaciones entre calidad de vida y productividad laboral, y entre calidad de vida y otros posibles factores tales como adherencia al cumplimiento.

(Este estudio ha sido patrocinado por Pfizer España, Madrid.) 


\section{Bibliografía}

1. Kuttner R. The American health care system--employer-sponsored health coverage. N Engl J Med 1999; 340: 248-52.

2. Antoñanzas F. Challenges to achieving value in drug spending in a decentralized country: the Spanish case. Value Health 2003; 6 (Suppl 1): 52-63.

3. Domínguez A, Inesta A. Establishing an insight-gaining area in pharmacoeconomics. Farm Hosp 2004; 28: 375-83.

4. Kaplan RM. The significance of quality of life in health care. Qual Life Res 2003; 12 (Suppl 1): 3-16.

5. Parkerson GR Jr, Hammond WE, Michener JL, Yarnall KS, Johnson JL. Risk classification of adult primary care patients by self-reported quality of life. Med Care 2005; 43: 189-93.

6. Manocchia M, Keller S, Ware JE. Sleep problems, health-related quality of life, work functioning and health care utilization among the chronically ill. Qual Life Res 2001; 10: 331-45.

7. Lerner DJ, Amick BC 3rd, Malspeis S, Rogers WH. A national survey of health-related work limitations among employed persons in the United States. Disabil Rehabil 2000; 22: 225-32.

8. Láinez JM, Domínguez M, Rejas J, Palacios G, Arriaza E, García-García $\mathrm{M}$, et al. Development and validation of the migraine screen questionnaire (MS-Q). Meadache 2005; 45: 1328-38.

9. Rejas J, Domínguez M, Láinez JM, Arriaza E, García-García M, Palacios $\mathrm{G}$, et al. Propiedades psicométricas del Índice de Impacto de la enfermedad en la Productividad Laboral (Indice IMPALA) en una población laboral. Revista Oficial de Medicina de Trabajo 2007 (aceptado para publicación).

10. Medical Outcomes Trust. Puntuación del Cuestionario de Salud SF-36. Versión española (España). Boston, MA: Medical Outcomes Trust, 1996.

11. Alonso J, Prieto L, Anto JM. The Spanish version of the SF-36 Health Survey (the SF-36 health questionnaire): an instrument for measuring clinical results. Med Clin (Barc) 1995; 104: 771-76.

12. Stewart WF, Lipton RB, Simon D. Work-related disability: results from the American migraine study. Cephalalgia 1996; 16: 231-38.

13. Alonso J, Regidor E, Barrio G, Prieto L, Rodriguez C, de la Fuente L. Population reference values of the Spanish version of the Health Questionnaire SF-36. Med Clin (Barc) 1998; 111: 410-16.

14. Alonso J, Ferrer M, Gandek B, Ware JE Jr, Aaronson NK, Mosconi P, et al. Health-related quality of life associated with chronic conditions in eight countries: results from the International Quality of Life Assessment (IQOLA) Project. Qual Life Res 2004; 13: 283-98.

15. Abell JE, Hootman JM, Zack MM, Moriarty D, Helmick CG. Physical activity and health related quality of life among people with arthritis. J Epidemiol Community Health 2005; 59: 380-85.

16. Gerson LB, Ullah N, Hastie T, Triadafilopoulos $G$, Goldstein $M$ Patient-derived health state utilities for gastroesophageal reflux disease. Am J Gastroenterol 2005; 100: 524-33.
17. Strine TW, Chapman DP, Kobau R, Balluz L, Mokdad AH. Depression, anxiety, and physical impairments and quality of life in the U.S. noninstitutionalized population. Psychiatr Serv 2004; 55: 1408-13.

18. Durham CF, Alden KR, Dalton JA, Carlson J, Miller DW, Englebardt $\mathrm{SP}$, et al. Quality of life and productivity in nurses reporting migraine. Headache 1998; 38: 427-35.

19. Watkins K, Connell CM. Measurement of health-related QOL in diabetes mellitus. Pharmacoeconomics 2004; 22: 1109-26.

20. Wang PS, Beck A, Berglund P, Leutzinger JA, Pronk N, Richling D, et al. Chronic medical conditions and work performance in the health and work performance questionnaire calibration surveys. J Occup Environ Med 2003; 45: 1303-11.

21. Lofland JH, Pizzi L, Frick KD. A review of health-related workplace productivity loss instruments. Pharmacoeconomics 2004; 22: 165-84.

22. Prasad M, Wahlqvist P, Shikiar R, Shih YC. A review of self-report instruments measuring health-related work productivity: a patientreported outcomes perspective. Pharmacoeconomics 2004; 22: 225-44.

23. Cote I, Farris K, Feeny D. Is adherence to drug treatment correlated with health-related quality of life? Qual Life Res 2003; 12: 621-33.

24. Carballo E, Cadarso-Suarez C, Carrera I, Fraga J, de la Fuente J, Ocampo A, et al. Assessing relationships between health-related quality of life and adherence to antiretroviral therapy. Qual Life Res 2004; 13: 587-99.

25. Shimbo T, Goto M, Morimoto T, Hira K, Takemura M, Matsui K, et al. Association between patient education and health-related quality of life in patients with Parkinson's disease. Qual Life Res 2004; 13: 81-9.

26. Jakobsson U, Hallberg IR, Westergren A. Overall and health related quality of life among the oldest old in pain. Qual Life Res 2004; 13: 125-36.

27. Kimura T, Ogushi Y, Takahashi M, Munakata Y, Ishii S. Association of health-related quality of life with health examination including organic functions and lifestyles in Japanese employees. Qual Life Res 2004; 13: 519-29.

28. Hesselink AE, Penninx BW, Schlosser MA, Wijnhoven HA, van der Windt DA, Kriegsman DM, et al. The role of coping resources and coping style in quality of life of patients with asthma or COPD. Qual Life Res 2004; 13: 509-18

29. Eklund M, Hansson L, Ahlqvist C. The importance of work as compared to other forms of daily occupations for wellbeing and functioning among persons with long-term mental illness. Community Ment Health J 2004; 40: 465-77.

30. Backman CL. Occupational balance: exploring the relationships among daily occupations and their influence on well-being. Can $\mathrm{J}$ Occup Ther 2004; 71: 202-9.

31. Chen WQ, Yu IT, Wong TW. Impact of occupational stress and other psychosocial factors on musculoskeletal pain among Chinese offshore oil installation workers. Occup Environ Med 2005; 62: 251-56. 